\title{
Taurine affects the predisposition to audiogenic epilepsy in PM rats
}

\author{
O.I. Prokudina ${ }^{1 *}$, T.A. Alekhina ${ }^{1}$, A.V. Solovyeva ${ }^{2}$ \\ ${ }^{1}$ Institute of Cytology and Genetics SB RAS, Novosibirsk, Russia \\ ${ }^{2}$ Altai State University, Barnaul, Russia \\ *e-mail: petrenko@bionet.nsc.ru
}

Key words: audiogenic seizure, epilepsy, taurine, animal model, PM rats

Motivation and Aim: Epilepsy is one of the most common serious neurological disorders, affecting about $1 \%$ of the world population. The searching for anticonvulsants and drugs for adjunctive therapy is required. PM rat strain had a high frequency of audiogenic seizure and can used as an animal model of some features of the human epilepsy. It was shown that PM rats had a lower taurine level in hippocampus [1]. Taurine (2-aminoethanesulfonic acid) is a natural amino acid with wide occurrence which play inhibitory role in the neural system. There are some works which show the decrease in predisposition to epilepsy after the taurine administration. The changed brain taurine level was demonstrated at the animal model of epilepsy [2]. The aim of this work was to investigate the potential effect of the taurine to predisposition to epilepsy in PM rats. Methods and Algorithms: The GC rat strain was used in the experiments. PM rat strain was breeding at the Institute of Cytology and Genetics (Novosibirsk, Russia) from a Wistar outbred population. Breeding rats for increased stereotypic hyperkinesis in the form of lateral rocking of the head and body (pendulum movements, PM) resulted in a heightened frequency of audiogenic epilepsy (about $80 \%$ ), whereas in the Wistar rats this frequency was about $25 \%$. Experiment was performed on adult male rats. Taurine (i.p. $800 \mathrm{mg} / \mathrm{kg}$ in saline) were given three times at $24 \mathrm{~h}$ intervals. After $1 \mathrm{~h}$ after last injections rats were tested for audiogenic seizures by exposing them to a sound of $109 \mathrm{~dB}$ intensity (90 sec duration) at special Plexiglas cube box. All procedures were carried out in accordance with the international guidelines for animal care and use (Ethical principles and guidelines for experiments on animals, Experientia 1995 Jan 15; 51(1):1-3). The results were statistically analyzed using Statistica 6.0.

Results: The acute taurine administration induced significant changes in seizure score severity in PM rats $(1.4 \pm 0.4$ vs. $2.58 \pm 0.15$ in control group, $p<0.001)$. No significant effect of the taurine administration on the post-ictal catalepsy, on the wild running time and on the latency time from audiogenic stimulus, onset to the initiation of wild running or clonus was observed.

Conclusion: The light anticonvulsive actions of taurine preliminary was shown in PM rats. The further research is required for determine the suitability of the taurine as a drug for adjunctive therapy.

Acknowledgements: Supported by the RFBR No. 17-04-01631-a.

\section{References}

1. Akulov A.E. et al. (2014). Selection for catatonic reaction in rats: a study of interstrain differences by magnetic resonance imaging. Zh Vyssh Nerv Deiat im I.P. Pavlova. 64(4):439-47.

2. Oja S.S., Saransaari P. (2013). Epilepsy Res. 104(3):187-94. 